\title{
Conceptos e ideas clave en la obra de Mary Ellen Richmond y la vigencia actual de su pensamiento
}

\author{
Key concepts and ideas in the work of Mary Ellen Richmond \\ and the currency of her thought
}

\author{
Bibiana TrAVI \\ Universidad Nacional de Luján $\square$ Argentina \\ bibiana.travi@gmail.com
}

Recibido: $19 / 05 / 2011$

Revisado: $19 / 05 / 2011$

Aceptado: 05/06/2011

Disponible on line: 29/09/2011

\begin{abstract}
Resumen
En este trabajo se presentan resultados de más de diez años de investigación histórico-disciplinar ${ }^{1}$. Su objetivo es dar visibilidad a conceptos e ideas clave desarrolladas por Mary Ellen Richmond, presentes en las obras Diagnóstico Social (1917) y Caso Social Individual (1922), y analizar su vigencia actual. Partimos de la hipótesis que el Trabajo Social desde su inicio ha planteado una serie de fundamentos teóricos que orientaron los modos de «comprender»y de «intervenir».

Sostenemos que ciertas nociones ampliamente utilizadas hoy en el campo disciplinar no son «nuevas», y que constituyeron la base del esquema teórico-conceptual articuladas entre sí en una coherente relación con una concepción de la ciencia «comprensivista», inspiradas en valores humanistas democráticos, el pragmatismo filosófico y el interaccionismo simbólico desarrollados principalmente por John Dewey y George H. Mead.

Los principales conceptos e ideas a analizar aquí son: las diferencias individuales, complejidad, diversidady apertura del yo. Sin embargo debido al entramado conceptual que presenta la autora, también se hará referencia a la relación teoría-práctica, la relación individuo-sociedad, la igualdad social y la contribución del Trabajo Social a la democracia, entre otros.
\end{abstract}

Palabras clave: conceptos, ideas, Richmond, diversidad, complejidad.

\begin{abstract}
This work presents the results of more than ten years of historical/professional research. Its objective is to cast light on concepts and ideas developed by Mary Ellen Richmond, which can be found in the books Social Diagnosis (1917) and What is Social Case Work (1922), and to analyze their current validity. We work from the hypothesis that, since its beginnings, Social Work has raised a series of theoretical concepts that have oriented the ways of «understanding» and of «intervening».

We argue that certain notions widely used in the professional field today are not «new», and that they have constituted the basis of the theoretical and conceptual framework articulated among them in a coherent relationship with a conception of the comprehensive social science, inspired in humanistic democratic values, the philosophical pragmatism and the symbolic interactionism developed mainly by John Dewey and George H. Mead.

The main concepts and ideas to be analyzed here are: individual differences, complexity, diversity and «the wider self $\gg$. Due to the conceptual framework presented by the author, however, this study will also refer to the relationship between theory and practice, the relationship between individual and society, social equality, and the contribution of Social Work to democracy, among others ${ }^{2}$.
\end{abstract}

Keywords: concepts, ideas, Richmond, diversity, complexity.

Referencia normalizada: Travi, B. (2011). «Conceptos e ideas clave en la obra de Mary Ellen Richmond y la vigencia actual de su pensamiento». Cuadernos de Trabajo Social, 24: 57-67.

${ }^{1}$ En el marco del Programa de Investigación en Trabajo Social, Dpto. de Ciencias Sociales, Universidad Nacional de Luján. Retoma como base los avances de la tesis del doctorado en Epistemología e Historia de la Ciencia (UNTREF) y la ponencia presentada por autora en el IV Encuentro de Investigadores en Trabajo Social (GIITS), Buenos Aires, 2011.

${ }^{2}$ Elaborado por el profesor Sebastián Seisdedos, Facultad de Lenguas, Universidad Nacional de Córdoba. 
Sumario: Introducción. 1. Presentación de la autora y sus obras. 1.1. Estructura y contenido de las obras Diagnóstico Social y Caso social individual. 2. Selección de conceptos e ideas presentes en las obras Diagnóstico social y Caso social individual. 3. Referencias bibliográficas.

\section{Introducción}

El objetivo de este artículo es dar visibilidad a conceptos e ideas clave desarrolladas por Mary Ellen Richmond, presentes en las obras Diagnóstico Social (1917) y Caso Social Individual (1922), y analizar su vigencia actual. Toda disciplina científica debe desarrollar de forma integral y articulada las dimensiones: epistemológica, teórico-conceptual-categorial, teórico-metodológica, técnico-instrumental-operativa y ético-política. Dimensiones que deben estar absolutamente integradas en cada intervención y momento del proceso metodológico y que, si bien cada una tiene su especificidad, el sobredimensionamiento de unas en desmedro de las otras empobrece el ejercicio profesional, impide el cumplimiento efectivo de los objetivos profesionales y atenta contra la construcción y fortalecimiento de la legitimidad de la profesión y sus profesionales.

En razón de ello, la habilitación para el ejercicio profesional requiere haber pasado por una instancia de formación debidamente acreditada. Dicha formación, de carácter teórico-práctico, tiene como uno de sus ejes centrales, el aprendizaje del proceso y desarrollo de la intervención profesional.

Sin embargo, según las diversas perspectivas y el signo de las épocas, en especial en América Latina, el Trabajo Social ha ido sobrevalorando una, en desmedro de las otras. En épocas de la reconceptualización, como bien lo expresa Mercedes Escalada (1986), se privilegiaron los objetivos de trasformación social (la «liberación del hombre oprimido»), en desmedro de la reflexión sobre el «qué», el objeto disciplinar. Se eliminaron de los planes de estudio los textos provenientes del mundo anglosajón y los marcos teórico-filosóficos estuvieron orientados principalmente por el materialismo histórico y dialéctico,

\footnotetext{
3 En términos de Jürgen Habermas.

4 En términos de Boaventura de Sousa Santos.
}

la pedagogía del oprimido de Paulo Freire y la teoría de la dependencia. Con el auge del desarrollismo se priorizaron «los métodos», siguiendo principalmente las «etapas de la planificación social» de un paradigma normativo. A fines de los ochenta, resurgió el interés por el desarrollo de propuestas teóricometodológicas y, en la última década del siglo XX en el Cono Sur, se impuso como tema casi excluyente en las publicaciones y los eventos científicos, la «cuestión social» y «el proyecto ético-político».

Paralelamente, aunque en menor medida, surge el interés y reflexión teórico-metodológica por la dimensión técnico-instrumental y, lentamente, con un peso absolutamente marginal en relación a las otras dimensiones, está surgiendo una preocupación por cuestiones de índole epistemológica y la reflexión sobre los conceptos y categorías que fundamentan la intervención profesional. Sin embargo, este fue un tema central en las preocupaciones de nuestras antecesoras.

Partimos de la hipótesis de que el Trabajo Social, desde su inicio, ha planteado una serie de fundamentos teóricos que han orientado los modos de «comprender» y de «intervenir» $\mathrm{y}$, que si bien una parte de estas nociones claves aún perdura como base conceptual, se ha producido un proceso de desvalorización, ocultamiento e invisibilización de su proceso de producción. Y si bien, algunos de estos conceptos aún tienen vigencia, hoy (re-)ingresan en América Latina en el Trabajo Social de la mano de autores como Bourdieu, Habermas o Giddens, negando, desconociendo su origen en el propio campo disciplinar y generando un ocultamiento de su producción en un doble proceso, que podríamos denominar, de «colonización interna $»^{3}$ y «epistemicidio disciplinar» ${ }^{4}$. 
Por lo tanto, este trabajo se propone develar que ciertas nociones, ampliamente utilizadas hoy en el campo disciplinar, no son «nuevas», que contienen una enorme riqueza conceptual y que constituyeron la base del esquema teórico-conceptual, articuladas entre sí en una coherente relación con una concepción de la ciencia "comprensivista», inspiradas en valores humanistas democráticos, el pragmatismo filosófico y el interaccionismo simbólico, desarrollados principalmente por John Dewey y George H. Mead.

\section{Presentación de la autora y sus obras}

Como sabemos, a raíz de la temprana muerte de sus padres, Mary Richmond fue criada por su abuela y sus tías en un ambiente de discusión sobre el sufragio femenino, la situación de la mujer, la discriminación racial, la religión, la política y diversos temas en debate en la sociedad norteamericana y «movimientos radicales» (Colcord y Mann, 1930, pp.15-16). Según sus propias palabras, fue su abuela quien le estimuló constantemente «a leer, a pensar por ella misma y a elaborar sus propios argumentos» (Bouquet, 2002). Durante su infancia y adolescencia desarrolló una verdadera pasión por la lectura que jamás abandonará. En tal sentido, Brigitte Bouquet sostiene: «en tanto lectora y escritora, ella ha tenido la preocupación por la palabra justa y la lucha contra la jerga profesional del Trabajo Social. En tal sentido, ella modificará regularmente las denominaciones que juzgue obsoletas».

A través de sus obras se observa la búsqueda permanente de aquellos términos rigurosos y precisos y que, a la vez, puedan dar cuenta simultáneamente de la filosofía y los principios que orientan al Trabajo Social. Una muestra en tal sentido se produce en 1918 , cuando convoca a un grupo de trabajadores sociales, representantes de diversos campos de actividad, para crear un «Comité de organización profesional» con el «doble objetivo de desarrollar una terminología y un código de ética» (op. cit.).

Con respecto a su producción escrita, según la recopilación de textos realizada por Colcord y Mann, se pueden contabilizar seis libros - uno en coautoría- y más de un centenar de artículos, conferencias, informes de gestión de su tarea en las $\mathrm{COS}^{5}$, editoriales en revistas especializadas e informes de investigación. Los temas más frecuentes están vinculados con la pobreza, la familia, la situación de las mujeres solas, el desempleo, el rol del Estado, la formación profesional y la interrelación entre el Trabajo Social y el movimiento reformista.

Aunque conocida principalmente por el desarrollo del Servicio Social de caso individual, reiterará hasta sus últimos días lo que considera una «verdad fundamental»: la «interdependencia existente entre la mejora individual y colectiva» y el necesario "avance conjunto de la reforma social y el trabajo social de casos» (Richmond, 2005, p. 425).

\subsection{Estructura y contenido de las obras Diagnóstico social y Caso social individual} De toda la producción desarrollada por las/os precursoras/es del Trabajo Social, entre fines del siglo XIX y principios del XX, estas dos obras son las más importantes ya que, por primera vez, sientan las bases y los fundamentos filosóficos y teórico-metodológicos de la disciplina. Asimismo, pueden ser consideradas como las obras de madurez en las que la autora expone, de forma clara, concreta y ordenada, su enorme conocimiento, sus reflexiones y la experiencia acumulada, ofreciendo un material de enorme riqueza, indispensable tanto para la formación como para los profesionales en ejercicio.

Social Diagnosis fue publicado en 1917 por la Russell Sage Foundation y traducido al español de forma completa por primera vez en el año 2005, por iniciativa del Consejo General de Diplomados de Trabajo Social y Asistentes Sociales de España. Hasta esa fecha, sólo se habían traducido algunos capítulos que se hallaban incorporados en otros textos.

Este texto tiene su origen quince años antes de su primera edición, ya que la autora tenía la «intención de brindar a los que recién se iniciaban una «explicación de los métodos que habían resultado útiles a sus antecesores» y la necesidad de explicitar las «semejanzas esenciales» entre las distintas formas que ad-

\footnotetext{
${ }^{5}$ Sociedad de Organización de la Caridad.
} 
quiere el Trabajo Social (p. XIX). Para su elaboración, llevó a cabo una minuciosa investigación, analizando 2.800 informes de casos provistos por 56 entidades sociales de tres ciudades diferentes, en las que se desarrollaban diversos tipos de Trabajo Social. Se trata de una sistematización de experiencias profesionales realizada medio siglo antes de que se produjeran en América Latina los primeros desarrollos teórico-metodológicos sobre la misma, entendiendo por sistematización la resignificación e indagación sobre la práctica profesional, el análisis de sus logros y dificultades, la evaluación de sus resultados en términos de intervención y la producción de nuevos conocimientos (Travi, 2006, p. 50).

La obra cuenta con un prefacio y veintiocho capítulos; tres apéndices, la bibliografía y el índice alfabético de los principales términos utilizados; en un total de 511 páginas ${ }^{6}$. El contenido central está dividido en tres partes: I.-Evidencia social, II.-Procesos que conducen al diagnóstico, III.-Variaciones en el proceso. En todos los capítulos se entrelazan la teoría y la experiencia, con referencias a ejemplos concretos y con numerosas notas al pie de página donde se cita bibliografía o a autores que merecen el respeto de la escritora. En todos los casos se observa una minuciosa rigurosidad en el uso y definición de los términos, lo que se apoya en argumentaciones acerca del porqué de su uso o elección. Con respecto a las referencias bibliográficas explica que, al no existir bibliografía sobre el objeto de la investigación, los títulos seleccionados son aquellos más cercanos y relacionados con el tema (2005, p. 587).

La finalidad que persigue es lograr una «mayor profesionalización», resalta que, además de la práctica, es fundamental el conocimiento teórico y que el «estudio de los procesos» de un campo disciplinar, el «conocimiento ordenado» no puede ser considerado como «enemigo de la inspiración»: el trabajador social «que prescinde de los precedentes de la técnica (...) deja tras de él un territorio completamente arrasado: el trabajador social que sólo se guía por la inspiración o el que acata estrictamente las normas y las formulas» (p. XXV).
Por último, cabe destacar el reconocimiento de la autora, por su apoyo y colaboración brindados, a renombradas figuras académicas de la Northwestern University, del Departamento de Historia de Vassar, la Universidad de Cambrigge (Massachussetts) y a los departamentos de investigación de las Escuelas de Educación Cívica y Filantrópica de Chicago, de Trabajo Social de Boston y de Filantropía de Nueva York, lo que permite dar cuenta del vínculo directo con los ámbitos y referentes de las ciencias sociales y humanas del momento, tal y como lo demuestra con claridad Miguel Miranda Aranda (2004).

La segunda obra en estudio, Caso social individual, traducida al español en los años 1960, es su libro más difundido en los países de habla hispana. Su título original What is Social Case Work?: an introductory description, formulado sugestivamente de forma interrogativa, constituye la primera producción teórica y el primer manual que se propone explícitamente como finalidad «buscar qué es el Servicio Social de Casos Individuales y por qué se recurre al mismo» (1993, p. 25). Las cursivas de la autora, reflejan claramente su preocupación respecto a la necesidad de una conceptualización sobre su naturaleza y su objeto (el qué) y de sus fundamentos o razón de ser (el porqué).

Para su elaboración tomó como base su propia experiencia profesional «completada por la lectura de numerosas observaciones sociales individuales», que fueron analizadas con absoluto rigor, explicitando claramente los criterios y procedimientos utilizados para su análisis, así como la definición de los principales conceptos y los autores que aportarán el sustento teórico desde diversos campos disciplinares (p. 60).

Si bien, como ella misma señalará, se trata fundamentalmente de un texto de carácter «descriptivo e introductorio», el valor incalculable de esta obra, no sólo radica en los aportes para la profesionalización del Trabajo Social, sino en el modo a través del cual la autora llega al desarrollo de sus ideas y a la capacidad de transmitirlas, de invitar al lector a reconstruir, desde las primeras inquietudes e interrogantes hasta los caminos elegi-

\footnotetext{
${ }^{6}$ Edición 1940
} 
dos o los autores de referencia consultados. En síntesis conjuga, una observación minuciosa, un pensamiento relacional y una «imaginación creativa».

El libro está compuesto por una introducción, seguida de diez capítulos y una conclusión. Los capítulos segundo y tercero se ocupan de describir y analizar con minuciosidad diferentes experiencias de colegas de entonces. Tal y como la autora señala, dichas experiencias se refieren a las intervenciones exitosas, es decir, que habían obtenido como resultado una verdadera transformación de la situación inicial. Luego propone una definición de Servicio Social de casos individuales, abocándose a los lineamientos metodológicos y al análisis de las características que el quehacer profesional toma en diferentes áreas específicas (hospitales, escuelas, familias, etc.). Los últimos dos capítulos están dedicados a explicitar y ejemplificar las «relaciones recíprocas» entre todas las formas del Servicio Social, culminando con la vinculación del Trabajo Social con la democracia, columna vertebral de su planteamiento. Finalmente presenta las conclusiones, en las que condensa los principios básicos del accionar del profesional y la filosofía que lo inspira, dejando claramente explicitada su perspectiva interaccionista, humanista y democrática y su convicción respecto al potencial del Trabajo Social para el logro de cambios duraderos.

\section{Selección de conceptos e ideas presentes en las obras Diagnóstico social y Caso so- cial individual}

Los principales conceptos y las ideas que se analizan en este artículo son: diferencias individuales, complejidad, diversidad y apertura del yo. Sin embargo, debido al entramado conceptual que presenta la autora, también se hará referencia a la relación teoría-práctica, la relación dinámica entre los diversos momentos del proceso de intervención y su concepción de la democracia, entre otros.

Cabe aclarar que, cuando hacemos referencia a las «ideas» lo planteamos, en términos de Lovejoy, como elementos constitutivos de un sistema. Para Dewey «una idea es una perspectiva, un marco de referencia, una categoría (en el sentido kantiano) donde los hechos y concepciones abstractas, la observa- ción y la intuición profunda forman una unidad» (citados en Nisbet, 2003, pp. 16-18).

Tomaremos, para iniciar el análisis, el capítulo decimonono de Diagnóstico social, en el que explicita y desarrolla la importancia de la relación teoría-práctica y presenta dos conceptos a los que considera como las bases y "presupuestos filosóficos (...). El primero está relacionado con las diferencias individuales, el segundo con la teoría de la "apertura del yo" ", ambos provenientes de la Psicología moderna, permiten clarificar «la diversidad humana» y su complejidad (2005, pp. 429-430, cursivas añadidas).

Antes de abocarse al desarrollo de los mismos, retoma y reitera los principales fundamentos y principios en los que se basa su concepción de Trabajo Social, entre ellos: la «interdependencia existente entre la mejora individual y colectiva» para fundamentar su postura respecto del necesario "avance conjunto de la reforma social y el trabajo social de casos» (p. 425).

Otro aspecto de constante preocupación para la autora es la relación y tensión existentes entre el avance del conocimiento científico, su «aplicación práctica» y los resultados de la misma en relación al primero. Con agudeza crítica observa que «el conocimiento actual no está siendo aplicado en el ámbito social porque no hemos logrado fomentar, en la medida en que sería necesario, una forma de trabajo social original y progresista entre los profesionales sociales» (p. 429).

De esta manera Richmond va a introducir dos categorías que luego el Trabajo Social «importará» o «reingresará» de las ciencias sociales de finales del siglo XX: la complejidad y la diversidad.

Siguiendo su línea argumental señala que, los logros en materia de reformas sociales, cada avance en Medicina, Psicología u otros campos, implican modificaciones mutuas en el diagnóstico y el tratamiento. De manera que no puede concebirse como social un «diagnóstico extremadamente breve» ni tampoco puede «una única reforma acabar con todos los males de la sociedad». Los trabajadores sociales que se conforman con una práctica de ese tipo «ignoran la complejidad, la gran diversidad de los materiales que tienen entre las manos» (p. 429). 
De esta breve, pero inequívoca afirmación, parte el análisis de las diferencias individuales. Hace referencia, en primer lugar, a «las características comunes de los seres humanos» y a su «importancia social», dado que «gracias a ellas, ha sido posible la mejora colectiva». Así vincula este tema con el desarrollo de las incipientes democracias que, si bien en un primer momento aplicaron «los mismos criterios a todos sus miembros» como garantía del principio de igualdad, prontamente se evidenció que, en aras de una mejora social, sería necesario «hacer cosas diferentes para y con personas diferentes» (p. 430). Con el fin de sustentar este posicionamiento apelará a los desarrollos teóricos de Edward L. Thorndike, Leonard P. Ayres y Paul H. Hanus, quienes coinciden en la importancia de la adaptación del sistema escolar y de las prácticas docentes a las necesidades individuales y locales.

El tema de las diferencias individuales, será profundizado en Caso social individual, donde intenta «ir más lejos», ampliar la mirada y relacionar el Trabajo Social con «otras tentativas conscientes, realizadas para adaptar al hombre a la vida social» $(1993$, p.85).

Con respecto a la teoría de la apertura del yo, expresa: «las diferencias individuales deben tenerse presentes en todos los ámbitos, pero la teoría de la apertura del yo, pese a que tiene lógicamente otras implicaciones, parece constituir la base del trabajo social de casos». Aquí se ve claramente una ruptura y un aporte novedoso en relación a las concepciones de una época en la que los trabajadores sociales iban dejando lentamente «clasificaciones generales» para "considerar al hombre globalmente», tomando conciencia de que «la mente humana (y, en realidad, la mente es el hombre) puede definirse como la suma de sus relaciones sociales» $(2005$, p. 431).

Para fundamentar esta «tesis» de corte claramente interaccionista, recurrirá nuevamente a Edward L. Thorndike y a Mark Baldwin, pero especialmente a Helen D. Bosanquet, una trabajadora social inglesa. Cita un texto de su obra The Standar of Life and Other Studies (1898), en el que expresa que el alma «es» o se conforma como producto de toda su experiencia y que en la medida que éstas sean po- sitivas «el yo crece y se expande (...) o se retrae cuando se suprime alguna esfera de actividad o un buen amigo nos deja», perdiendo literalmente «una parte de nosotros mismos».

Apelando esta vez a la literatura argumentará, a partir de una de las máximas de Polonio $^{7}$, que «un hombre es verdaderamente sus compañías que frecuenta más las que frecuentaron sus antepasados» (p. 431), incorporando por último, una visión del cambio como algo permanente, inevitable y «como una de las condiciones de la salud» ya sea de signo positivo o negativo.

Con el desarrollo de estos conceptos puede advertirse su visión precursora, dinámica e integral del hombre como producto y productor de sus relaciones sociales y de su entorno, con capacidad de modificarse y modificarlo y, por otra parte, el papel que le otorga a la teoría en la intervención profesional.

Si bien la autora era consciente de las grandes limitaciones del Trabajo Social de la época, también era optimista en cuanto a sus potencialidades. Así consideraba que, los nuevos desarrollos que aportaba la ciencia -agrega que el Trabajo Social «ya había experimentado exitosamente en Inglaterra con la labor de Octavia Hill»-implicarían nuevas exigencias y competencias profesionales para el estudio de las diferencias humanas, su comprensión, la implementación de medidas eficaces y la obtención de resultados exitosos que, en términos de la autora, se resumen en el mejoramiento del nivel y calidad de vida de los más desprotegidos.

En tal sentido, serán las relaciones sociales de los individuos afectados las principales herramientas para utilizar en su rehabilitación. Más adelante concluye: «si queremos que los resultados de nuestra labor sean satisfactorios, tendremos que hacer cosas diferentes con y para personas diferentes, y estudiar sus diferencias» (p. 433). Para abordarlo es necesario ampliar la mirada y dirigirla hacia el grupo familiar del sujeto, sus vínculos, el entorno, e ir «más allá de la estrecha percepción que el cliente tiene sobre su situación, así como del reducido círculo que forman nuestras propias predisposiciones y procedimientos preferidos» (p. 171). Advierte tam-

\footnotetext{
${ }^{7}$ Personaje de Hamlet.
} 
bién que el estudio del hombre como «un todo» debe corresponderse con métodos y procesos que no se reduzcan a cuestiones técnicas, sino que se sometan «a un todo más amplio. A partir del estudio de ese todo $-\mathrm{y}$ no insistiendo solamente en la técnica-, podremos adquirir el conocimiento necesario para analizar correctamente las situaciones individuales» (p. 433).

Si bien la influencia del Pragmatismo y el Interaccionismo Simbólico, a través de las figuras de John Dewey y George Mead, es clara a lo largo de toda su obra, es en relación al tema de las relaciones sociales y la constitución social del yo donde se observa con mayor claridad. Ejemplo de ello es la adopción de un sistema teórico-filosófico y una visión no dicotómica de la relación individuo-sociedad, en particular la influencia del medio ambiente y la perspectiva de un sujeto capaz de transformarse a sí mismo y a la vez a su entorno, adelantándose medio siglo a quienes, en los años 1960, plantearán la interacción sujeto-estructura, las dimensiones materiales y subjetivas, y los complejos procesos de la construcción social de la realidad ${ }^{8}$. Sus aportes serán fundamentales en relación a sus teorías sobre la participación democrática como valor universal y la unidad del conocimiento, cuya premisa central es justamente la unidad intrínseca entre teoría y práctica; es decir, que el «conocimiento está inseparablemente unido al hacer», así como el valor que atribuye a la «experiencia» y sus novedosas «doctrinas pedagógicas».

En los capítulos, «La interdependencia humana» $\mathrm{y}$ «Las características individuales», de Caso social individual manifiesta su deseo de ir más allá de las definiciones elaboradas e intentar «relacionar el servicio social de casos individuales con otras tentativas conscientes, realizadas para adaptar el hombre a la sociedad» (1993, p. 85).

Con respecto a «la relación individuo-sociedad» señala:

No desprecies a ninguna criatura humana. Todos los hombres están hechos de la misma manera, lo que ha servido para formar a la hu- manidad en general. El mundo infinito se refleja en el microcosmo. Ya que quieres que todos marchen contigo hacia la gran aurora, ayuda a ese hombre (Johan Bojer, 1923 citado en Richmond, 1993, p. 84).

Como ya se hizo referencia, para la autora el Trabajo Social debe estar orientado por una filosofía que permita dar respuestas a su razón de ser y sus fines últimos, sin descuidar «el elemento humano que es la materia de su trabajo» (p. 84). Así, criticará a las asistentes sociales que «preconizan una forma de tratamiento social para las personas que se encuentran por debajo de lo que ellas llaman "las fronteras de la miseria" y de otro modo, verosímilmente superior, para aquellos que están por encima de este nivel» (p. 85), argumentando que los médicos no practican una medicina para pobres y otra para ricos. Con el fin de aportar nuevas ideas respecto de estas formas de «estratificación social» apela a una frase de Thomas Huxley:

Me pregunto a veces si la gente que habla con tanta desenvoltura de eliminar a los ineptos, ha considerado alguna vez, sin apasionamiento su propio caso. Hay que ser perfecto para no recordar que en una o dos oportunidades a nosotros también nos hubieran podido fácilmente clasificar de ineptos (Tomas Huxley, 1894, citado en Richmond, 1993, p. 85)9 .

Lamenta que, en su caso personal, el trabajo diario haya provocado una disminución en su interés por la filosofía, pero que retoma gradualmente a partir de ciertas «revelaciones». En el texto se observa claramente una problematización y ruptura con sus primeras creencias basadas en «la concepción romántica que hace del individuo una suerte de "caballero solitario"», que "había caído en la trampa del contrato social» y por lo tanto debía «protegerse lo mejor posible de los ataques del mismo» (p. 86). Las teorías de las que se vale para interpelar estas viejas concepciones son nuevamente los avances de la Psicología y la Psicología social: James Mark Baldwin, Josiah Royce y George Mead.

8 Entre otros P. Bourdieu y J. Habermas.

9 Huxley, Thomas (1894). Evolution and Ethic, (citado en Edwin C. Concklin (1921). The Direction of Human Evolution. p. 39). 
De Baldwin y Royce toma fundamentalmente sus aportes sobre: el desarrollo y las fases de la personalidad y su evolución como necesidad para adaptarse a la sociedad; la importancia del ambiente y del medio; sus estudios de la ontogénesis y el concepto de herencia social. De Royce: el proceso de desarrollo de la consciencia de sí mismo como parte del proceso de socialización.

Sin embargo, el autor de mayor influencia para Mary Richmond es George Mead quien «va más lejos todavía cuando afirma que la sociedad no es solamente el medio por el cual se desarrolla la personalidad, sino también la fuente y origen de ésta». Según la autora, la «teoría del yo ampliado» (...) es una de las piedras angulares del Servicio Social de Casos Individuales». A través de estas ideas plantea:

[La] necesidad de desembarazarnos de los últimos vestigios de esta concepción que nos obsesiona todavía y que hace residir la inteligencia del hombre en alguna parte de su cabeza o en un lugar cualquiera del espacio. La constitución mental del hombre está formada por la suma de sus dones naturales y de las experiencias y las relaciones sociales que ha tenido hasta ese momento (p. 87).

Otro autor al que hace referencia es el filósofo y discípulo de Royce, William Ernest Hocking, para quien las «fuerzas constructivas conscientes» cuentan más que la herencia, y que si bien «la naturaleza puede completar a otras criaturas, la criatura humana debe completarse a sí misma» (op.cit., p. 88).

Esta novedosa concepción para la época tiene numerosas implicaciones para la intervención profesional, ya que la mentalidad $h u-$ mana, lejos de ser «fija» o «inalterable», está en un permanente proceso de cambio y «es infinitamente sensible a las sugestiones, es capaz de recibir poderosas impresiones de afuera, formar nuevas costumbres, aprovechar las ocasiones que se presentan, asimilar tanto el bien como el mal» (p. 87).

Tratados estos temas, se aboca nuevamente al análisis de las características y diferencias individuales, retoma su defensa de la $d i$ versidad, la igualdad social y la relación del trabajo social con la construcción de una sociedad democrática.
Como ya se hizo referencia, para la autora, el Servicio Social de casos individuales se desarrolla a partir del estudio de las relaciones del individuo para lograr su «readaptación», teniendo en cuenta de forma conjunta y recíproca tanto el «espíritu del cliente» como el «ambiente», sin posibilidades de trazar una línea demarcatoria que defina qué aspecto corresponde a la herencia y cuáles se deben a las influencias del medio. Las habilidades, capacidades, la idiosincrasia u otros aspectos hacen que cada sujeto se diferencie de sus semejantes y «todas estas circunstancias influyen a su vez sobre el medio social por el cual el cliente estará ulteriormente influenciado». Citando al profesor MacIver, afirmará que «sociabilidad e individualidad son dos aspectos de una misma realidad» (p. 98).

Luego de haber analizado estas cuestiones desde una perspectiva fundamentalmente filosófica, y frente al predomino en la época de concepciones «innatistas» o que ponen el énfasis en la «herencia» como determinante de la personalidad, apelará a los biólogos, a los eugenésicos, a psicólogos y a sociólogos para fundamentar su posicionamiento.

Se apoya en los estudios biológicos que demuestran el papel fundamental que desempeñan el ambiente y la educación en el desarrollo del hombre, así como en la relación intrínseca entre estos factores y la herencia, y las diferencias sustanciales en relación a este último factor, si se trata de animales o de seres humanos. Así, toma partido en el debate sobre «la importancia relativa de la herencia y del medio como factores del desarrollo humano» (p. 99), citando al Dr. Myerson, quien cuestiona las leyes de Mendel en el sentido que no pueden aplicarse a la «herencia humana». Pone en duda los hallazgos de los eugenésicos ya que considera que confunden «herencia física»y «herencia social», que son muy diferentes (op. cit.).

Finalmente, encuentra en la obra Our Social Heritage, del socialista Fabiano Graham Wallas, una explicación convincente acerca de la importancia de la herencia social en todas las especies cuyas crías permanecen largo tiempo con sus padres, y la herencia social como factor de supervivencia. Incorpora el concepto de «hábito» para dar cuenta del im- 
pacto permanente que producen sobre el hombre las tradiciones sociales y para establecer una diferencia con la inalterabilidad del «plasma germinativo» ${ }^{10}$ (p. 100). "Además de esta herencia, tenemos que contar también los efectos que produce la educación, la religión, el gobierno y las relaciones sociales sobre la existencia del individuo, libre de sus movimientos y que participa de la vida social». Una perspectiva que considera esperanzadora frente a los pronósticos sombríos de los eugenésicos (p. 100).

Ahora bien, reconoce que los aportes de la eugenesia son importantes para reconocer un dato esencial: que las diferencias individuales existen. La cuestión es: ¿cómo se aborda esta situación en una sociedad democrática? ¿Cuál debería ser el rol del Estado y cuál el papel del Trabajo Social?

Plantea que un sistema democrático debe tener en cuenta la gran diversidad existente entre los seres humanos, no sólo «las diferencias heredadas e invariables, sino también (...) todas las disimilitudes provenientes de la diversidad, de nuestras impresiones en el curso de nuestra vida social, así como de la forma diferente de reaccionar de cada uno a la misma impresión» (p. 100).

Por lo tanto, se opone a toda forma de Estado autocrático y critica uno de los principales rasgos de la política tradicional estadounidense: el principio de «lo mismo para todos». Considera que implica una visión equivocada acerca de la igualdad «como equivalente a parecido, en el sentido de identidad» (en términos de Félix Adler), y no en el sentido de unas similitudes sobre las cuales sobresalen las disimilitudes deseables» (pp. 100-101). Así argumenta:

Decir que todo hombre es igual a sus semejantes, significa que cada uno tiene los mismos derechos a volverse diferente de los otros, a adquirir una personalidad distinta, a proyectar su propio rayo en el haz de los diversos colores cuya combinación forma la luz blanca de la vida espiritual (Adler, citado en Richmond, 1993, p.101).

Sobre el tema de la contribución del Servicio Social de casos individuales a la demo- cracia, la igualdad y los rasgos autocráticos de la política tradicional, citará el debate y discurso de Gertrude Vaile en las Conferencias Nacionales de Servicio Social de 1915 y 1918 (p. 101).

Siguiendo a Platón, considera que «la esencia de la igualdad consiste en tratar las cosas desiguales en forma desigual». Aquí pone en juego la relación entre «la semejanza humana» y las «variaciones entre los grupos». Por ejemplo, los inmigrantes chinos pueden ser vistos como si fueran todos iguales, debido a la propia ignorancia e incapacidad de revelar «la diversidad infinita de los dones y de las características» de cada uno en particular (p. 102). Haciendo referencia al trato recibido por los inmigrantes «como si pertenecieran a una clase aparte» (op. cit.) señala que sólo después de haber penetrado en el conocimiento de su realidad profunda, y de reconocer en ellos las variaciones individuales y sus semejanzas fundamentales con todos los hombres, se impone «la necesidad de ser guiados en su derecho a ser colocados en condiciones favorables, a un desarrollo completo, a la diversidad» (op. cit.).

Asimismo se adelanta casi un siglo a la noción de «discriminación positiva», defendiendo ciertas garantías legales para las mujeres en la industria, que algunas feministas pretendían suprimir luego de haber accedido al sufragio universal, argumentando que no se puede confundir la igualdad con la uniformidad (op. cit., p. 102).

Con respecto a la relación entre las diferencias individuales, la democracia y la igualdad social, retoma nuevamente a «un radical como Grahamm Wallas, quien exhorta «a la democracia a que reconozca plenamente las diferencias individuales». En relación con la educación, el autor considera que, a medida que los medios de evaluación psicológica sean más completos, las diferencias que separan a los niños se basarán más en la «naturaleza» que en la alimentación de niño rico o pobre, de su medio culto o simple, y así, el progreso social se basará en el «reconocimiento de las diferencias individuales»» (pp. 102-103).

${ }^{10}$ Desarrollado por el biólogo alemán, August Weismann, 1834-1914. 
Estas consideraciones tienen una implicación clara y directa en la intervención profesional, ya que,

Nos revelan las dos fases de un programa realmente democrático: por una acción colectiva inteligente, este programa iguala las probabilidades de éxito de todos; por otra parte, reconoce la diversidad, estableciendo normas de administración pública que cada una realiza sin cesar; cosas diferentes para personas diferentes y por personas diferentes (p. 103).

Así, el Trabajo Social va avanzando progresivamente desde concepciones basadas en «uniformidades rígidas», advirtiendo que si bien las clasificaciones entre grupos - «desocupados», «madres solas» o «inmigrantes recientes»-- pueden ser de utilidad, no deben ser transformadas en un fin. Cuestiona así severamente «las locuras de la “americanización"» que consisten en tratar a todos los inmigrantes como «semejantes». Considera que «ningún programa uniforme puede aplicarse con éxito a todos los miembros» de un mismo grupo (por ejemplo, los desocupados). Por lo tanto es fundamental reconocer sus capacidades en cuanto a trayectoria profesional, su nivel de calificación y experiencias $\operatorname{previas}^{11}$ (p. 104).
Señala los avances que van realizando los trabajadores sociales en el reconocimiento de la «maravillosa diversidad que, destacándose desde el fondo de nuestra naturaleza común, reina en toda agrupación social» y que éste es el principio que debe imponerse como «elemento unificador» de todo programa de atención de necesidades sociales. Sin embargo sólo un trabajador social que cuente con una gran habilidad para descubrir los matices los podrá aplicar (p. 104).

Implica que no puede haber una aplicación mecánica de «remedios sociales», sino que es necesaria una investigación de las diferencias que subyacen en cada agrupación social. Así, el Trabajo Social va dejando atrás las «fórmulas desabridas» para dar lugar a análisis más profundo de las situaciones sociales y de los individuos con los que trabaja, pero debe esforzarse por «desarrollar la técnica especial» que el abordaje de las complejas y diversas situaciones exige.

Para concluir y sintetizar entonces su posicionamiento, recurrimos a la cita de una de sus exalumnas: «Pienso que el Servicio Social de Caso Individual vive y crece del mismo modo que la democracia, y posee en sí el poder de efectuar una revolución. En efecto no puede existir una verdadera democracia sin este servicio» (p. 105).

\section{Referencias bibliográficas}

Agnew, E. N. (2004). From Charity to Social Work. Mary E. Richmond and the Creation of an American Profession. Illinois: University of Illinois Press.

Bouquet, B. (2002). «Préface à la deuxième édition». En M. Richmond, Les méthodes nouvelles d'asistence. Le service social de cas individuels. París: École Nationale de la Santé Publique.

Colcord, J. C. Mann, R.Z.S. (1930). The long view. Papers and Adresses by Mary E. Richmond. Nueva York: Russell Sage Foundation. Reimpreso en 1970.

Escalada, R. M. (1986). Crítica a los métodos de la reconceptualización del Trabajo Social. Tegucigalpa: Guaymuras.

Kisnerman, N. (1998). Pensar el Trabajo Social. Buenos Aires: Espacio.

Miranda Aranda, M. (2004). De la caridad a la Ciencia. Pragmatismo, interaccionismo simbólico y Trabajo Social. Zaragoza: Mira.

Nisbet, R. (2003). La formación de pensamiento sociológico. Buenos Aires: Amorrortu. Tomo I.

Richmond, M. (1940). Social Diagnosis. Nueva York: Russell Sage Foundation, (1 ${ }^{\mathrm{a}}$ ed. 1917).

Richmond, M. (1993). Caso Social Individual. Buenos Aires: Humanitas, (1 ${ }^{\text {a }}$ ed. 1922, Russell Sage Foundation, New York).

${ }^{11}$ La revalorización de las trayectorias individuales hoyo es planteado por Robert Castel y Pierre

Rosanvallon entre otros. 
Richmond, M. (2005). Diagnóstico Social. Madrid: Siglo XXI Editores de España (1 ${ }^{\mathrm{a}}$ ed. 1917, Russell Sage Foundation, Nueva York).

Travi, B. (2006). La dimensión técnico-instrumental en Trabajo Social. Reflexiones y propuestas acerca de la entrevista, la observación, el registro y el informe social. Buenos Aires: Espacio. 